\title{
The early development of medial coronoid disease in growing Labrador retrievers: radiographic, computed tomographic, necropsy and micro-computed tomographic findings
}

\begin{abstract}
Medial coronoid disease (MCD) encompasses lesions of the entire medial coronoid process (MCP), both of the articular cartilage and the subchondral bone. To detect the earliest signs of $\mathrm{MCD}$, radiography and computed tomography were used to monitor the development of MCD in 14 Labrador retrievers, from 6 to 7 weeks of age until euthanasia. The definitive diagnosis of MCD was based on necropsy and micro-computed tomography findings. The frequency of MCD in the dogs studied was 50\%. Radiographic findings did not provide evidence of MCD, ulnar subtrochlear sclerosis or blunting of the cranial edge of the MCP. Computed tomography was more sensitive $(30.8 \%)$ than radiography $(0 \%)$ in detecting early MCD, with the earliest signs detectable at 14 weeks of age. A combination of the necropsy and micro-computed tomography findings of the MCP showed that MCD was manifested as a lesion of only the subchondral bone in dogs <18 weeks of age. In all dogs (affected and unaffected), there was close contact between the base of the MCP and the proximal radial head in the congruent joints. Computed tomography and micro-computed tomography findings indicated that the lesions of MCD probably originated at the base of the MCP.
\end{abstract}

Keyword: Canine; Labrador retriever; Medial coronoid process; Micro-computed tomography 